


VOLUME 45 . NO.4. DECEMBER 2020



$E D|Z| O N|\cdot M| N E R \vee A \cdot M E D \mid C A$ 


\title{
Codon bias analyses on thyroid carcinoma genes
}

\author{
Daniele PEPE *, Kim DE KEERSMAECKER
}

Department of Oncology, Laboratory for Disease Mechanisms in Cancer, Katholieke Universiteit (KU) Leuven, Leuven, Belgium

*Corresponding author: Daniele Pepe, Department of Oncology, Laboratory for Disease Mechanisms in Cancer, Katholieke Universiteit (KU) Leuven, 3000 Leuven, Belgium. E-mail: daniele.pepe@kuleuven.be

\section{A B S T R A C T}

BACKGROUND: Thyroid carcinoma is one of the most common cancers in the world. Although the genetics of thyroid carcinoma was intensively studied, new mechanisms could be involved in its development as the codon bias. In this paper, we studied the codon bias of thyroid-cancer genes, considering not only the sequences but also the synonymous mutations.

METHODS: Different measures and statistical analyses were employed to characterize the thyroid-cancer genes. We considered classical measures as RSCU and ENC, the compositional and protein characteristics, but also the codon bias landscape via the \%MinMax algorithm.

RESULTS: The compositional analyses highlighted two groups of thyroid cancer genes according to the GC\% and GC $3 \%$ content. The ENC did not show a clear codon bias in the genes. Differently, the RSCU analyses showed interesting codons that could play an important role in the development of thyroid cancer as the codon Ser-tcG. Furthermore, interesting synonymous mutations were detected that could affect the codon bias. The codon bias landscape detected genes enriched in rare codons as AKAP9 and KTN1. A cluster analysis based on \%MinMax classified the thyroid cancer genes in four different groups according to the distribution of rare/frequent codons in the sequence.

CONCLUSIONS: This is the first study that analyzed the codon bias in thyroid cancer genes based also on synonymous mutations. This study provided different hints that should be further investigated by wet-lab validation and that it could open new scenarios in the understanding the molecular mechanisms involved in thyroid cancer development based on codon bias.

(Cite this article as: Pepe D, De Keersmaecker K. Codon bias analyses on thyroid carcinoma genes. Minerva Endocrinol 2020;45:295-305. DOI: 10.23736/S0391-1977.20.03252-6)

KEY WORDS: Codon usage; Silent mutation; Thyroid neoplasms.

$\mathrm{T}$ hyroid carcinoma (TC) is one of the most common cancers in the world and it is the most common endocrine malignancy. The incidence worldwide is about $2.1 \%$ of all cancer diagnoses, with about $77 \%$ occurring in women. ${ }^{1,2}$ Nowadays TC is efficiently diagnosed by techniques that can detect also small lesions in the thyroid gland such as computed tomography, ultrasonography and magnetic resonance imaging. However, such sensitivity could lead to overdiagnosis, i.e. diagnosis of thyroid lesions that do not lead to death or to the manifestation of symptoms. ${ }^{3}$ TC is divided in two main subtypes: follicular-derived thyroid cancers and medullary TC. There are two types of follicular derived thyroid cancers: 1) differentiated thyroid cancer that accounts for more than $95 \%$ of cases and it includes papillary thyroid cancer, follicular thyroid cancer and Hurthle cell thyroid cancer; 2) anaplastic thyroid cancer, a rare form of TC. ${ }^{4}$ Medullary TC is uncommon, accounting for $1-2 \%$ of all TCs. It originated in the parafollicular neuroendocrine cells of thyroid and not in the follicular epithelial cells as the first subtype. Concerning the genetics, one of the most mutated signaling pathway is the mitogen-activated protein kinase (MAPK). It has a key role in the regulation of cellular proliferation and in 
the transmission of growth signals from plasma membrane to nucleous. ${ }^{4}$ The genetics is different between the two types of thyroid cancer. In the follicular-derived thyroid cancers the most frequent point mutation is BRAF V660E. Furthermore, mutations in the RAS family of oncogenes and in TERT as well as translocations inducing PAX8-peroxisome proliferator-activated receptor $\gamma$ (PPAR $\gamma$ ) and the RET-papillary thyroid cancer family fusions have been observed. Concerning medullary thyroid cancer, mutations in the RET proto-oncogene are the most frequent. ${ }^{4}$ An overlooked biological mechanism that could help to better characterized the development and regulation of TC is the codon usage bias (CUB). As well known, the genetic code is degenerated, and 18 out of 20 amino acids are encoded by more than one codon defined as synonymous codons. The usage of synonymous codons is not uniform in coding sequences (CDS) and this leads to CUB. The different usage of synonymous codons plays an important role in controlling different cellular processes as gene and protein expression, protein folding, translation efficiency. ${ }^{5}$ Interestingly, there are good examples that illustrate how the usage of particular synonymous codons can have an important functional role. ${ }^{6,7}$ For example, the three RAS family proteins KRAS, NRAS and HRAS with an important role in tumorigenesis share $85 \%$ of amino acid identity, whereas the expression of those proteins can be quite different. In one study, the expression of KRAS and HRAS was compared and KRAS protein levels turned out to be significantly lower than HRAS. Analysis of mRNA sequences revealed that the third position of the codons used in KRAS is enriched for $\mathrm{A} / \mathrm{T}$, corresponding to rare codons, while this position in HRAS is enriched for $\mathrm{G} / \mathrm{C}$. The conversion of rare to common codons of KRAS sequences by oncogenic mutations such as ttA to $\mathrm{ctG}$, ttT to $\mathrm{ttC}$, agA to $\mathrm{cgC}$ and aaA to aaG increased the expression and the tumorigenicity. ${ }^{6}$ This example shows how much the codon bias and synonymous mutations (SMs) can affect the tumorigenicity of genes. In this paper, we evaluated the codon bias of the thyroid cancer genes by several measures commonly used in the evaluation of CUB as for instance the effective number of codons (ENC) and the relative synonymous codon usage (RSCU). 8,9 Those measures however do not allow evaluating locally biased distributions of rare and frequent codons along the sequence (codon landscape). To this end, we employed the \%MinMax algorithm. ${ }^{10}$ Furthermore, we analyzed the CUB based on codons subjected to mutational events to evaluate: 1) which codons are more subjected to SMs; 2) which mutations could have an impact on CUB, generating rare or more frequent synonymous codons compared to the corresponding wildtype codons. The results of this study will allow to better understand the CUB in thyroid cancer genes and will offer the possibility to detect possible SMs that could have a functional impact in the pathogenesis of TC.

\section{Materials and methods}

All the analyses reported in this paper were performed by R software version 3.6.3.11

\section{Thyroid cancer gene detection}

The Catalogue of Somatic Mutations in Cancer (COSMIC) Cancer Gene Census (CGC) offers a description of the genes driving human cancer. ${ }^{12}$ At the moment, 719 cancer genes are present in this dataset. All 30 genes associated to thyroid were considered. The codon sequences of these genes were downloaded using the $\mathrm{R}$ package biomaRt. ${ }^{13}$ For each gene, the longest transcript with several nucleotides multiple of 3 and with no missing nucleotides was selected. The transcripts for which no sequence was available were discarded.

\section{Human codon frequencies}

The codon usage table was obtained from hive. biochemistry.gwu.edu/review/codon. ${ }^{14}$

\section{Synonymous mutations in TC genes}

We extracted the SMs linked to TC genes from the MC3 dataset provided by the Multi-Center Mutation Calling in Multiple Cancers project that generated a comprehensive encyclopedia of somatic mutation calls for the TCGA data to enable robust cross-tumor-type analyses. ${ }^{15}$ 


\section{Compositional properties}

The overall nucleotide composition $(\mathrm{A} \%, \mathrm{C} \%$, $\mathrm{T} \%$ and $\mathrm{G} \%$ ) and nucleotide composition in the third codon position, overall GC content and $\mathrm{GC}$ content at the first (GC1), second (GC2) and third positions (GC3) were used to perform a PCA analysis to evaluate if there are compositional differences in the thyroid cancer genes sequences.

\section{Relative synonymous codon usage (RSCU)}

RSCU (Relative synonymous codon usage) values are the observed frequency at genome level of a synonymous codon divided by the expected frequency obtained assuming a uniform synonymous codon usage (i.e. all the codons for a given amino-acid have the same probability). The RSCU is 1 if there is no codon bias, less than 1 if the observed codon is less frequent than expected and greater that 1 if the codon is more frequent than expected. In agreement with previous papers, $8,9,16$ we refer to synonymous codons with RSCU values $>1.6$ and $<0.6$ as over-represented and under-represented, respectively.

The RSCU values were computed for the codons of each thyroid cancer gene sequence, for the codons subjected to SMs, and for the codons after the mutational events. About the last two analyses, the goal is to understand if there is a codon bias in the codons that are mutated and in the corresponding mutated codons. The significant RSCU will be compared between the three groups. The assumption is that if the SMs are passenger mutations, the RSCU values between the original sequences and the codons subjected to mutations should be comparable. The SMs were extracted from the MC3 dataset at pancancer level, to evaluate the general tendency of those genes to be mutated.

\section{Correspondence analysis}

Correspondence analysis (COA) is used to examine the major trend in the variation of codon usage pattern among genes and distributes the codons in axis 1 and axis 2 based on these trends. The R packages "FactorMiner" and "factoextra" were employed for the COA.
Effective number of codons (ENC) and neutrality plot

The ENC (effective number of codons) measure evaluates to what degree all 61 codons are used in a gene. ${ }^{17,} 18$ The measure goes from a minimum value of 20 to a maximum of 61 . When the ENC value of a gene is less than or equal to 35 , a relevant bias exists. ${ }^{9}$ To evaluate if the codon usage is affected by mutation and selection the ENC-GC3\% plot was determined where the ENC values are plotted as the ordinate and the $\mathrm{GC} 3 \%$ as the abscissa. If most of the points fall near the expected curve than mutation is the main force that could shape codon usage. ${ }^{17}$ The neutrality plot verifies whether the mutation bias is the main force that characterizes the codon usage. There is a mutation bias if the correlation between the GC12 and GC3 is statistically significant and the slope of the regression line is close to 1.19

\section{Properties of protein}

Grand Average of Hydrophobicity (GRAVY) index of amino acid sequences was computed as proposed by Kyte et al. ${ }^{20}$ A positive GRAVY score indicates hydrophobic characteristic of a protein, while a negative score indicates a hydrophilic nature of the protein.

Aromaticity value is the percentage of aromatic amino acids (Phe, Tyr, Trp) present in a protein.

\section{The \%MinMax algorithm}

The \%MinMax algorithm was previously described. ${ }^{10}$ For the $\mathrm{J}_{\text {th }}$ codon of the $\mathrm{I}_{\text {th }}$ amino acid with $\mathrm{n}$ synonymous codons, \%MinMax calculates the difference between the usage frequency of the actual codon $\mathrm{X}_{\mathrm{ij}}$ and the average codon usage frequency for all $\mathrm{n}$ synonymous codons encoding that amino acid $\left(\mathrm{X}_{\mathrm{avg}, \mathrm{i}}\right)$. In case the difference is positive, it is divided by the difference between the maximum $\left(\mathrm{X}_{\max , \mathrm{i}}\right)$ and the average, otherwise by the difference between the average and the minimum $\left(\mathrm{X}_{\min , \mathrm{j}}\right)$. In this way, if the codon usage of the codon $\mathrm{J}_{\text {th }}$ is less than the average, you have a negative value indicating the rareness of the codon, whereas a positive value indicates how frequent the codon is. The $\%$ MinMax range 
is between -1 and +1 . The first one is obtained when the codon is the least frequent for a certain amino acid and +1 when it is the most frequent.

To have an idea if a gene is dominated by the presence of frequent or rare codons, we have summed the \%MinMax values for each codon and normalized according to the number of the codons in the gene. Furthermore, we divided the sequences of each gene in 10 equal parts going from the 5' part of the coding sequence to the 3' part and analyzed the trend of codon usage bias using the \%MinMax in each of these 10 regions. This allowed to verify if there are regions that are more subjected to codon bias.

\section{Results}

\section{Thyroid cancer genes}

30 genes associated to thyroid cancer were detected. They are: $A K A P 9, B R A F, C C D C 6$, CDC73, DICER1, EIF1AX, ERC1, GOLGA5, HMGA1, HOOK3, KRAS, KTN1, MEN1, MTOR, NCOA4, NRAS, NTRK1, PAX8, PCM1, PPARG, PRKARIA, RET, STRN, TFG, TPM3, TPR, TRIM27,TRIM33, TSHR, ZNF331. The SMs associated to those genes were extracted from the MC3 dataset. 1228 SMs were detected involving 754 samples and 28 tumor types.

\section{Compositional properties}

Two types of measures were computed to evaluate the nucleotide composition of each thyroid cancer gene sequence: 1) the nucleotide composition on the entire sequence $(\mathrm{A} \%, \mathrm{C} \%, \mathrm{~T} \%$, and $\mathrm{G} \%$ and $\mathrm{GC} \%)$; 2) the composition at codon level (A3\%, C3\%, T3\%, and G3\%), GC content at the first $(\mathrm{GC} 1 \%)$, second $(\mathrm{GC} 2 \%)$ and third positions (GC3\%) of each codon.

These measures were then used to perform a PCA analysis to verify if there are clear differences in the nucleotide composition between the cancer genes. The first two components were considered as they explained the $88.81 \%$ of the total variance. A correlation analysis between the first two components and the variables revealed a high positive correlation of the first component with the overall $\mathrm{GC} \%$ and $\mathrm{GC} 3 \%$ content (0.99 and 0.97 respectively) and a high negative

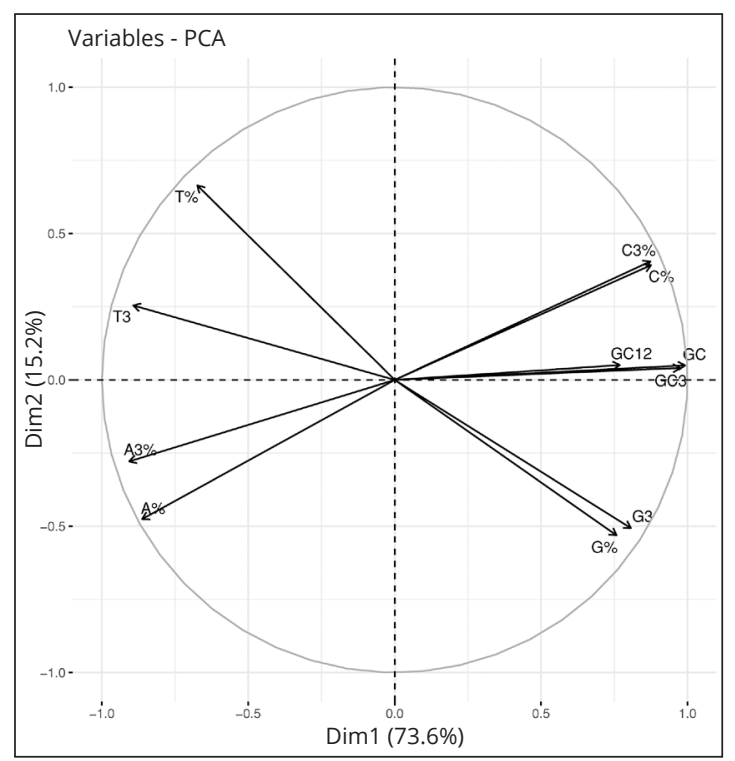

Figure 1.-Contribution of compositional properties on the first two PCA components.

correlation with $\mathrm{A} 3 \%$ and $\mathrm{T} 3 \%$ (-0.86 and 0.89 respectively). The highest positive and negative correlations for the second component were observed with overall T\% (0.66) and overall G\% $(-0.53)$ (Figure 1). The first two components were used to perform a hierarchical clustering based on Euclidean distance. The best number of clusters was found using the silhouette method. Two clusters showed the highest silhouette value (0.56) and are shown below. It seems that what characterized the two clusters is the first component (Figure 2). Therefore, the clusters are distinguished by the $\mathrm{GC} \%$ and $\mathrm{GC} 3 \%$ content, where the genes in the cluster constituted by PAX8, MTOR, TRIM27, RET, CCDC6, MEN1 and NTRK1 resulted in a higher GC\% and $\mathrm{GC} 3 \%$ content.

\section{RSCU analyses}

The RSCU allows to determine the codons that are over- or underrepresented in the thyroid cancer gene sequences, codons subjected to mutations and mutated codons. We considered a codon as overrepresented if the average RSCU is greater than 1.6, and underrepresented if the average RSCU is less than 0.6. There are four amino acids that are enriched in all the three groups: arginine, serine, isoleucine and glutamine. 


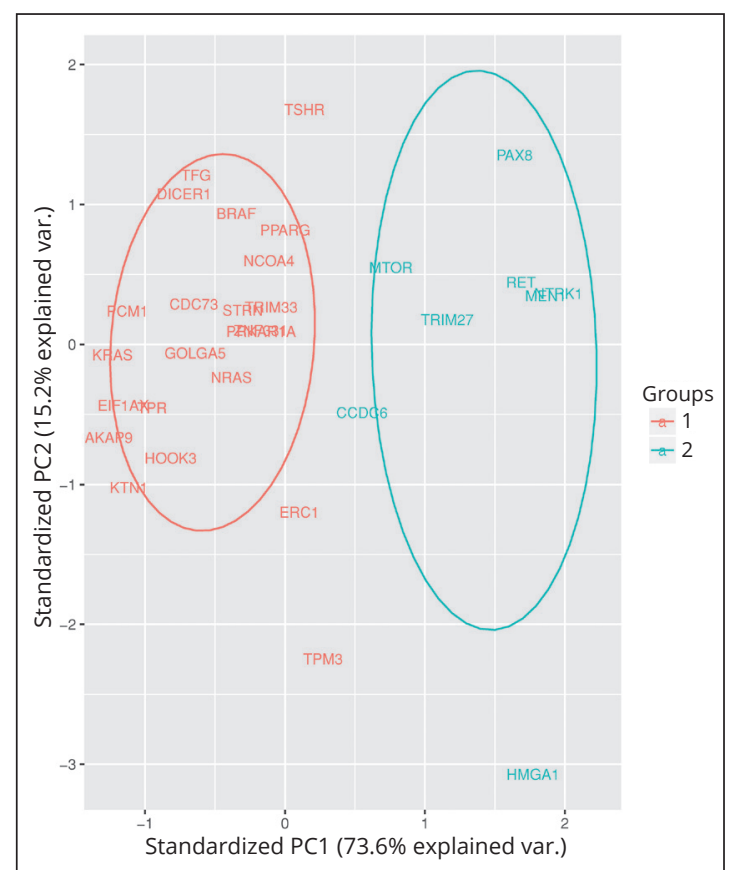

Figure 2.-The two gene clusters detected on the first two PCA components based on the compositional properties of TC gene sequences.

To facilitate the interpretation, a plot with the relative frequencies of the enriched codons is reported (Figure 3).

The frequencies are computed at amino-acid level by dividing the frequency of each codon by the frequency of the codon with the highest frequency in the human protein coding genome.

The enriched codons in the three categories are showed in the Figure 4.

The overrepresented codons in the gene sequences are Arg-Aga and Leu-ctg. They are also the codons with the highest frequency at aminoacid level (Figure 3). The underrepresented codons Thr-acG, Ile-atA, Gln-caA, Pro-ccG, ArgcgT, Leu-ctA, Ala-gcG, Ser-tcG are also the least frequent codons at amino-acid level (Figure 3). It appears therefore that the frequency of the codons in the thyroid gene sequences is not different from the frequency of the codons in the human protein coding genome.

Concerning the RSCU values of the codons subjected to SMs, there are 8 overrepresented and 10 underrepresented codons (Figure 4). The overrepresented were Ile-atC, Arg-cgG, Leu-ctC, Leu-ctG, Val-gtG, Ser-tcC, Ser-tcG, Phe-ttC.

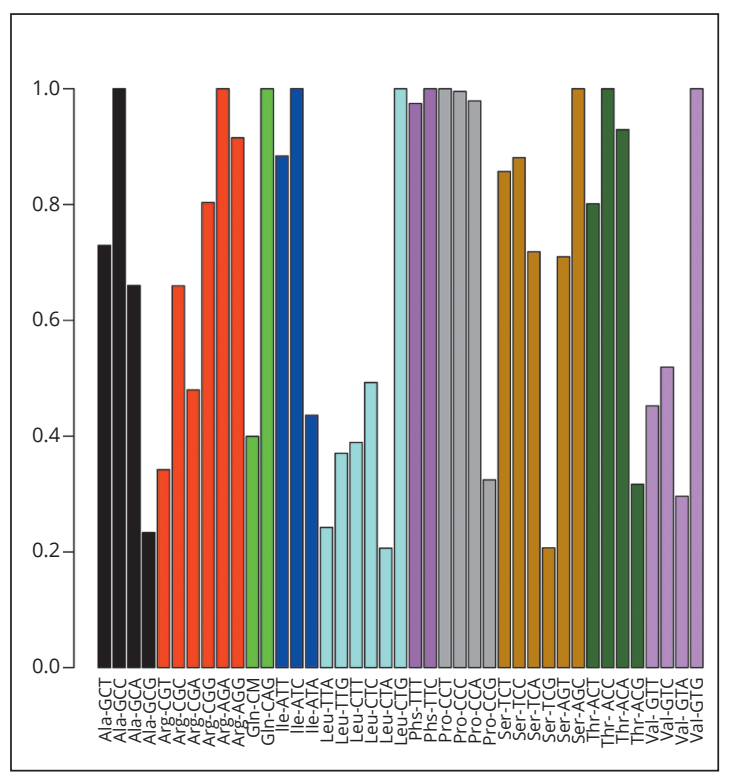

Figure 3.- Relative frequencies of the enriched codons based on RSCU values.

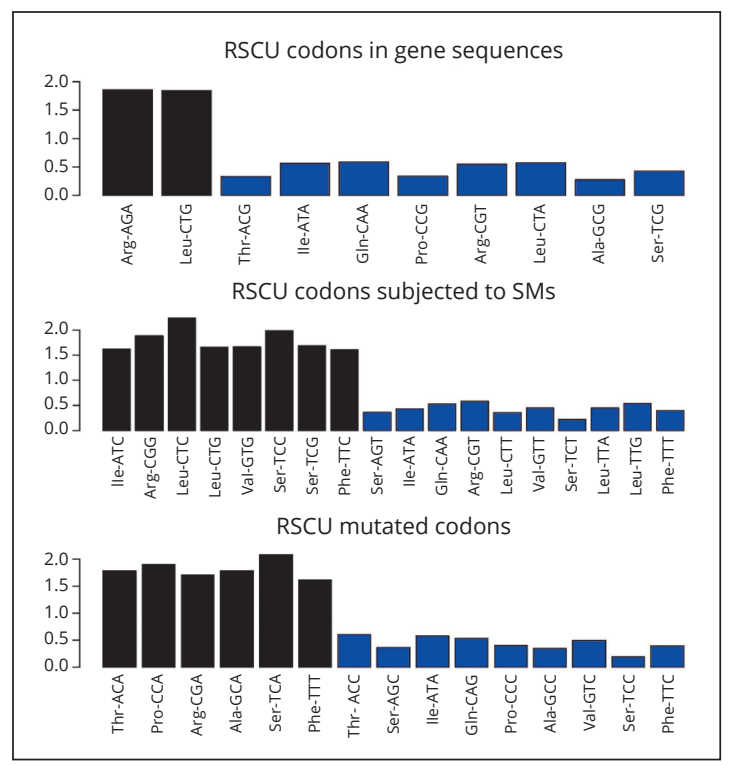

Figure 4.-Over/under-represented codons based on gene sequences, on codons subjected to mutations and mutated codons.

They are also the most frequent at amino-acid level. The only exception is Ser-tcG that is one of the codons that tends to be more subjected to SMs, but it is also the least frequent among the Serine codons. The Serine codon agT, the least frequent after tcG, is at least 3.4 times more 
frequent. The Ser-tcG could be part of a possible mechanism of regulation to activate cancer genes. In fact, the decoding time of codons depends on the corresponding tRNA availability. Recent studies showed that rare codons with less abundant cognate tRNAs are decoded slower and this mechanism can affect translation elongation rates. ${ }^{21,22}$ The genes with the highest tcG RSCU values on codons subjected to SMs are: $C C D C 6$, EIF1AX, HMGA1 and ZNF331. The underrepresented codons were Ser-agT, Ile-atA, Gln-caA, Arg-cgT, Leu-ctT, Val-gtT, Ser-tcT, Leu-ttA, Leu-ttG, Phe-ttT. There are two underrepresented codons that have the highest frequency at transcriptome level Ser-agT and Ser-tcT. Both involve the amino-acid serine. The fact that they are overrepresented in the sequence and have a low tendency to be mutated could mean that they have a key role in these genes, or they are in highly conserved regions of the genes.

To make easy the interpretation of the enriched mutated codons, a table reporting the occurrences of the mutated codons and the corresponding codons subjected to SMs is reported (Table I).

When analyzing the over-represented mutated codons, we noticed that the codon Thr-acA occurs 44 times and in 35 cases is generated from the low frequent codon Thc-acC. Pro-ccA has a frequency of 33 and 23 times is generated from the low frequent codon ccG. Concerning Arg$\operatorname{cg} \mathrm{A}$, the tendency is the opposite as it is a low frequent codon generated from high frequent codons as $\operatorname{cgG}$ and $\operatorname{cgC}$.

About Ala-gcA occurs 39 times and 29 from

TABLE I.-The relative frequencies of the most represented mutations are reported together with their occurrences and the corresponding amino-acid.

\begin{tabular}{|c|c|c|c|c|c|c|}
\hline Mutation & Mut_freq & Wt_cod & Mut_cod & Freq_wt & Freq_mt & AA \\
\hline $\mathrm{gcG} / \mathrm{gcA}$ & 29 & $\mathrm{gcG}$ & $\mathrm{gcA}$ & 0.00603 & 0.01705 & Ala \\
\hline $\mathrm{gcC} / \mathrm{gcA}$ & 5 & $\mathrm{gcC}$ & $\mathrm{gcA}$ & 0.02584 & 0.01705 & Ala \\
\hline $\mathrm{gcT} / \mathrm{gcA}$ & 5 & gcT & $\mathrm{gcA}$ & 0.01885 & 0.01705 & Ala \\
\hline $\mathrm{gcT} / \mathrm{gcC}$ & 5 & $\mathrm{gcT}$ & $\mathrm{gcC}$ & 0.01885 & 0.02584 & Ala \\
\hline $\mathrm{gcA} / \mathrm{gcC}$ & 2 & $\mathrm{gcA}$ & $\mathrm{gcC}$ & 0.01705 & 0.02584 & Ala \\
\hline $\mathrm{gcG} / \mathrm{gcC}$ & 2 & $\mathrm{gcG}$ & $\mathrm{gcC}$ & 0.00603 & 0.02584 & Ala \\
\hline $\mathrm{cgG} / \mathrm{Cga}$ & 15 & $\mathrm{cgG}$ & Cga & 0.0107 & 0.00639 & Arg \\
\hline $\mathrm{cgC} / \mathrm{Cga}$ & 4 & $\operatorname{cgC}$ & $\mathrm{Cga}$ & 0.00878 & 0.00639 & Arg \\
\hline Aga/Cga & 3 & Aga & Cga & 0.01332 & 0.00639 & Arg \\
\hline $\operatorname{cgT} / \mathrm{Cga}$ & 1 & $\operatorname{cgT}$ & $\mathrm{Cga}$ & 0.00456 & 0.00639 & Arg \\
\hline $\mathrm{caA} / \mathrm{caG}$ & 11 & $\mathrm{caA}$ & $\mathrm{caG}$ & 0.01415 & 0.03543 & Gln \\
\hline atC/atA & 8 & atC & atA & 0.01867 & 0.00814 & Ile \\
\hline atT/atA & 3 & at $\mathrm{T}$ & at $\mathrm{A}$ & 0.0165 & 0.00814 & Ile \\
\hline $\mathrm{ttC} / \mathrm{ttT}$ & 55 & $\mathrm{ttC}$ & $\mathrm{ttT}$ & 0.01753 & 0.01708 & Phe \\
\hline $\mathrm{ttT} / \mathrm{ttC}$ & 3 & $\mathrm{ttT}$ & $\mathrm{ttC}$ & 0.01708 & 0.01753 & Phe \\
\hline $\mathrm{ccG} / \mathrm{ccA}$ & 23 & $\mathrm{ccG}$ & $\operatorname{ccA}$ & 0.00619 & 0.01867 & Pro \\
\hline $\mathrm{ccC} / \mathrm{ccA}$ & 6 & $\mathrm{ccC}$ & $\operatorname{ccA}$ & 0.01898 & 0.01867 & Pro \\
\hline $\mathrm{ccT} / \mathrm{ccA}$ & 4 & ccT & $\mathrm{ccA}$ & 0.01907 & 0.01867 & Pro \\
\hline $\mathrm{ccG} / \mathrm{ccC}$ & 1 & $\mathrm{ccG}$ & $\mathrm{ccC}$ & 0.00619 & 0.01898 & Pro \\
\hline $\mathrm{ccT} / \mathrm{ccC}$ & 1 & ccT & $\mathrm{ccC}$ & 0.01907 & 0.01898 & Pro \\
\hline $\mathrm{tcG} / \mathrm{tcA}$ & 28 & tcG & tcA & 0.00408 & 0.01415 & Ser \\
\hline $\mathrm{tcC} / \mathrm{tcA}$ & 11 & tcC & tcA & 0.01735 & 0.01415 & Ser \\
\hline agT/agC & 8 & $\operatorname{agT}$ & $\operatorname{agC}$ & 0.01398 & 0.0197 & Ser \\
\hline $\mathrm{tcT} / \mathrm{tcC}$ & 3 & tct & tcC & 0.01688 & 0.01735 & Ser \\
\hline $\mathrm{tc} A / \mathrm{tcC}$ & 1 & tcA & $\mathrm{tcC}$ & 0.01415 & 0.01735 & Ser \\
\hline acG/acA & 35 & $\mathrm{acG}$ & $\mathrm{acA}$ & 0.00564 & 0.01654 & Thr \\
\hline $\mathrm{acC} / \mathrm{acA}$ & 9 & $\mathrm{acC}$ & $\mathrm{acA}$ & 0.0178 & 0.01654 & Thr \\
\hline $\mathrm{acT} / \mathrm{acC}$ & 8 & acT & $\mathrm{acC}$ & 0.01426 & 0.0178 & Thr \\
\hline $\mathrm{acA} / \mathrm{acC}$ & 2 & $\mathrm{acA}$ & $\mathrm{acC}$ & 0.01654 & 0.0178 & Thr \\
\hline $\mathrm{acT} / \mathrm{acA}$ & 1 & acT & $\mathrm{acA}$ & 0.01426 & 0.01654 & Thr \\
\hline $\mathrm{acG} / \mathrm{acC}$ & 1 & $\mathrm{acG}$ & $\mathrm{acC}$ & 0.00564 & 0.0178 & Thr \\
\hline gtG/gtC & 4 & gtG & gtC & 0.0259 & 0.01344 & Val \\
\hline $\mathrm{gtA} / \mathrm{gtC}$ & 1 & gtA & $\mathrm{gtC}$ & 0.00767 & 0.01344 & Val \\
\hline $\mathrm{gtT} / \mathrm{gtC}$ & 1 & gtT & $\mathrm{gtC}$ & 0.01172 & 0.01344 & Val \\
\hline
\end{tabular}


the low frequent codon Ala-gcG. The same trend was observed for Ser-tcA (39 times frequent, 28 out of 39 from the low frequent codon tcG).

Concerning the under-represented mutated codons, Ile-atA occurs 11 times and all the times from a high-frequent codon. Gln-caG occurs 11 times starting from the low frequent wild-type codon Gln-caA.

Correlation analysis did not show a significant correlation between the RSCU values of gene sequences and the codons subjected to SMs (Kendall's tau $=-0.08, \mathrm{P}=0.35$ ) and the RSCU values of gene sequences and mutated codons (Kendall's tau $=0.098, \mathrm{P}=0.27$ ).

The comparison between the enriched codons in the gene sequences and the enriched codons subjected to SMs revealed four interesting codons: Leu-ctG, Ile-atA, Gln-caA and Ser-tcG. The first three are over-represented in both. This means that probably the highest frequency of those codons in the sequences render them more subjected to mutations.

\section{COA on RSCU values from gene sequence}

A COA was performed on the RSCU values obtained from TC genes (Figure 5A, B). The first two components explained most of the variance (60.4\%, first one $50.1 \%$ and the second $10.3 \%$ ).

To understand the meaning of the first and second component, correlation analyses were performed considering the compositional gene sequence information, gene expression and properties of the proteins. The first component showed a very high correlation with $\mathrm{GC} \%(0.96)$ and GC3\% (0.99). The genes with the highest squared cosine $(\cos 2)$ similarity with the first component were RET (0.92), MEN1 (0.88), NTRK1 (0.88), PCM1 (0.84), AKAP9 (0.82) and KTN1 (0.80) (Figure 5A). Regarding the codons, the highest $\cos 2$ were observed for Ile-atC (0.85), Glu-aaA (0.84), Glu-gaG (0.84) (Figure 5B).

The second component showed the highest correlation with the GRAVY index (0.32), the G\% (-0.34) and C\% (0.30). At codon level, the highest $\cos 2$ were observed for Ser-tcG $(0.55)$ and Val-gtA (0.54) (Figure 5B). Instead the genes with the highest $\cos 2$ resulted $\operatorname{EIF} 1 A X(0.53)$ and PRKAR1A (0.3) (Figure 5A).

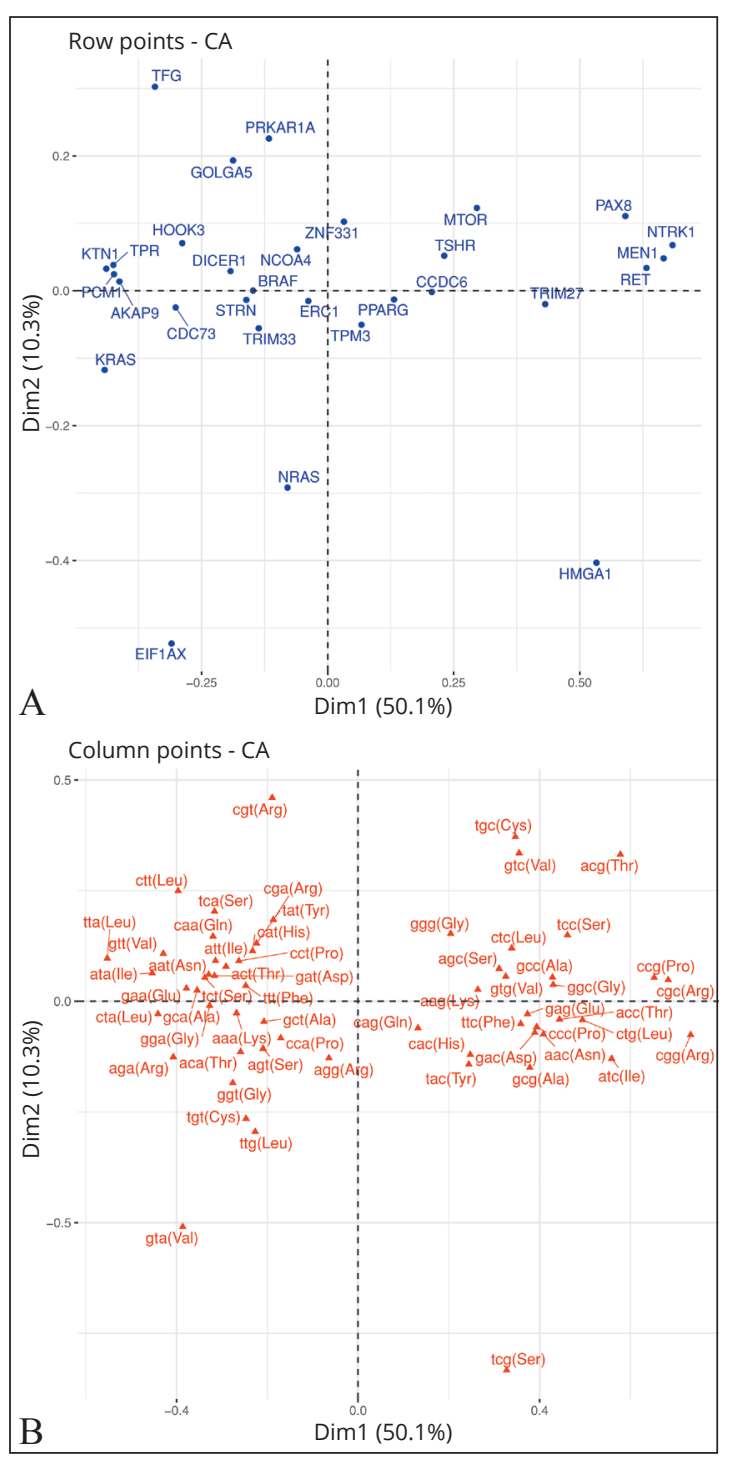

Figure 5.-A)Distribution of genes based on the CA on the RSCU values computed on the gene sequences; B) distribution of codons based on the CA on the RSCU values computed on the gene sequences.

\section{Effective number of codons and neutrality plot}

The effective number of codons (ENC) analysis did not show genes with a clear codon bias usage as the lowest observed value was 41.66. The genes with the lowest ENC were MEN1, NTRK1, PAX8 and RET (41.66, 41.67, 43.65, 43.75 respectively) (see "ENC thyroid cancer genes" in Figure 6). This result confirms the RSCU correspondence analysis where those genes had the highest contribution to the definition of the first 


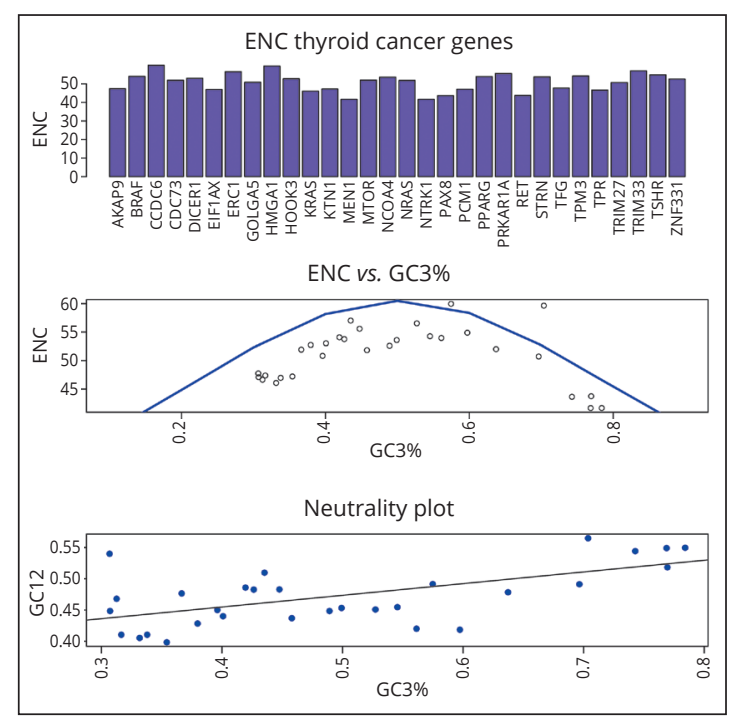

Figure 6.-ENC values for each thyroid cancer gene; ENC vs. GC3\% and neutrality plot.

component. We evaluated also if the ENC values correlate with the hydrophobicity and aromaticity of the proteins measured respectively by the GRAVY and aromaticity score. A previous paper suggested that hydrophobicity and aromaticity could affect the CUB. The correlation Spearman analysis did not show a significant correlation nor with the GRAVY score $(\mathrm{P}=0.35)$ nor with the aromaticity score $(\mathrm{P}=0.25)$. Furthermore, the plot ENC-GC3\% was evaluated (see "ENC vs. GC3\%" in Figure 6). Most points followed the expected ENC curve (visualized in blue), indicating that the codon usage is mainly influenced by mutational pressure. This is also confirmed by the neutrality plot that analyzes the correlation and the linear regression between $\mathrm{GC} 12 \%$ and $\mathrm{GC} 3 \%$ (see "Neutrality plot" in Figure 6). The regression analysis between $\mathrm{GC} 12 \%$ and $\mathrm{GC} 3 \%$ showed a significant intercept $(0.38, \mathrm{P}<0.00001)$ and a significant $\beta$-coefficient (coefficient $0.19, \mathrm{P}=0.0003$ ).

The Spearman test revealed a significant correlation between $\mathrm{GC} 3 \%$ and $\mathrm{GC} 12 \%$ with a value of $0.54(\mathrm{P}=0.003)$. This indicates the presence of mutation bias as force that characterizes the codon usage.

\section{MinMax results}

For each gene, the MinMax algorithm was applied. The average on MinMax values was computed for each gene to verify if there is the predominance of frequent or rare codons in the genes (Figure 7A).

The HGMA1, MEN1, PAX8, NTRK1 and RET have the highest average MinMax values indicating a high presence of the most frequent codons in the sequence. The genes with the highest presence of rare codons in their sequence are $A K A P 9$ and KTN1.

To understand the distribution of the codon bias based on the MinMax algorithm, each gene sequence was divided in 10 equal parts going from the 5' part to the 3' part of the coding sequence and the average MinMax values for each of these parts were computed.

A hierarchical clustering was performed based on the Euclidean distance and the Ward method to determine if there are common codon patterns along the thyroid gene sequences (Figure 7B). The best number of clusters was four as indicated by the silhouette analysis.

Cluster one consists of 13 genes, cluster two of 14 , the third by one and the fourth by two. To understand the differences, a line plot illustrating the MinMax value distribution is shown in Figure $7 \mathrm{C}$. The different codon bias trends were visualized by computing the average of the \%MinMax values for each part and for each cluster. The distribution of the MinMax values along the sequences revealed different patterns. Gene cluster number 3 represented by the gene TRIM27 showed an average of the MinMax values close to zero in the $6^{\text {th }}, 8^{\text {th }}$ and $10^{\text {th }}$ part and a rich presence of highly frequent codons in the rest of the gene sequence. This means that the frequency of the codons in these areas is close to the average frequency at amino-acid level. The cluster number 1 and 2 showed an almost uniform distribution of the MinMax values along the sequences. The cluster 4 instead is the one with the highest presence of high frequent codons above all in the first $7^{\text {th }}$ and $10^{\text {th }}$ part of the sequences.

\section{Discussion}

The codon bias is due to the redundancy of the genetic code. This implies that there are amino acids represented by more than one codon. This redundancy can be used as a key regulatory 




Figure 7.-A) \%MinMax distribution on gene sequences; B) dendrogram showing the three clusters obtained by \%MinMax analysis; C) \%MinMax values for each cluster show different pattern of rare codons. mechanism in the expression of genes. It was observed a correlation between the frequency of a synonymous codons and the corresponding tRNA availability. ${ }^{5}$ Highly expressed proteins are enriched in high frequent synonymous codons in the genome recognized by abundant charged tRNAs. The codon bias was highly overlooked as possible mechanism to induce the development of cancer. In this study, we performed several analyses to characterize the genes associated to $\mathrm{TC}$, although they play an important pathogenesis role also in other tumors types. The analysis based on the compositional properties revealed two different sets mainly characterized by the $\mathrm{GC} \%$ and $\mathrm{GC} 3 \%$. For example, the genes $M E N 1$ and NTRK1 showed a high content of GC\% and $\mathrm{GC} 3 \%$ while the genes $K R A S$ and $A K A P 9$ are characterized by $\mathrm{A} \%$ and $\mathrm{A} 3 \%$ content (Figure $2,3)$. The observed codon bias in the TC genes is mainly due to mutational pressure as it was confirmed by the neutrality plot, showing a significant correlation between $\mathrm{GC} 12 \%$ and $\mathrm{GC} 3 \%$, and by the ENC-GC3\% plot. The ENC analysis did not show a clear bias in the number of used synonymous codons. The minimum value was observed for the gene MEN1 with a value of 41.66. The RSCU analyses permitted to discover different important information about the codons in the TC gene sequences, the codons subjected to SMs and the mutated codons. The correlation test between the RSCU based on gene sequences and the RSCU based on codons subjected to SMs was not significant. This means that the frequency of the observed codons in the sequences is not correlated with the mutational frequency of the corresponding codons. The comparison between the enriched codons in those two groups highlighted codons that could have a pathogenetic role. A very interesting example was the codon Ser-tcG that resulted to be one of the codons more subjected to SMs but also the least frequent among the Serine codons. Other two Serine codons as Ser-agT and Ser-tcT were under-represented in the codons subjected to SMs but also highly frequent at genome level. Comparing the frequencies of the enriched mutated codons with the corresponding codons before the SMs, we observed that there are mutations that cause the passage from high frequent synonymous codons 
to low frequent synonymous codons and viceversa. For example, we observed that there was a mutational pressure to transform the low frequent codon Thc-acC in the high frequent codon Thr-acA. For the mutated codon Arg-cgA the tendency is the opposite as it is a low frequent codon generated from high frequent codons as Arg-cgG and Arg-cgC. The \%MinMax revealed confirmed the results obtained by the compositional properties and the COA analysis showing genes with a high presence of frequent codons as HGMA1, MEN1, NTRK1 and RET and genes enriched in rare codons as $A K A P 9$ and KTN1. The clustering analysis, based on the \%MinMax values computed dividing the gene sequences in 10 parts, showed 4 different clusters, so 4 different codon usage patterns of the synonymous codons along the gene sequences.

\section{Conclusions}

In conclusion, this study is the first one to consider the codon bias in TC genes considering not only the gene sequences but also the pattern of SMs in those genes. Recent papers ${ }^{23,} 24$ described how SMs could play a role as driver mutations. So far, those mutations were excluded in the mutational analyses considered mainly as passenger mutations. The genes described in this paper were associated to cancer in the COSMIC Cancer Gene Census but based on studies that excluded the potential role of SMs and codon bias. We detected interesting SMs that could affect the codon bias of those genes in cancer pathogenesis. Further validation analyses are required to confirm the potential role of those SMs. We also offered a general picture of codon bias in TC genes by several parameters as ENC, \%MinMax and RSCU. Considering the results illustrated in this paper, we hope to arouse interest in the possible role of codon bias and SMs in the study of TC.

\section{References}

1. Ferlay J. SoerjomatAram I, Ervik M, Dikshit R, Eser S, Mathers C., et al. GLOBOCAN 2012: Estimated Cancer Incidence, Mortality and Prevalence Worldwide in 2012 v1.0. IARC CancerBase No. 11; 2015 [Internet]. Available from: https://publications.iarc.fr/DatAbases/Iarc-Cancerbases/
GLOBOCAN-2012-Estimated-Cancer-Incidence-MortalityAnd-Prevalence-Worldwide-In-2012-V1.0-2012 [cited 2020, Oct 26].

2. Kitahara CM, Sosa JA. The changing incidence of thyroid cancer. Nat Rev Endocrinol 2016;12:646-53.

3. VacCArella S. Franceschi S, Bray F, Wild CP, Plummer M, Dal Maso L. Worldwide Thyroid-Cancer Epidemic? The Increasing Impact of Overdiagnosis. N Engl J Med 2016;375:614-7.

4. Cabanillas ME, McFadden DG, Durante C. Thyroid cancer. Lancet 2016;388:2783-95.

5. Quax TE, Claassens NJ, Söll D, van der Oost J. Codon Bias as a Means to Fine-Tune Gene Expression. Mol Cell 2015;59:149-61.

6. Lampson BL, Pershing NL, Prinz JA, Lacsina JR, Marzluff WF, Nicchitta CV, et al. Rare codons regulate KRas oncogenesis. Curr Biol 2013;23:70-5.

7. Ali M, Kaltenbrun E, Anderson GR, Stephens SJ, Arena $\mathrm{S}$, Bardelli A, et al. Codon bias imposes a targetable limitation on KRAS-driven therapeutic resistance. Nat Commun 2017;8:15617.

8. Uddin A, Paul N, Chakraborty S. The codon usage pattern of genes involved in ovarian cancer. Ann N Y Acad Sci 2019;1440:67-78.

9. Uddin A, Chakraborty S. Codon Usage Pattern of Genes Involved in Central Nervous System. Mol Neurobiol 2019;56:1737-48.

10. Clarke TF 4th, Clark PL. Rare codons cluster. PLoS One 2008;3:e3412.

11. R Core Team. R: A language and environment for statistical computing; 2020 [Internet]. Available from: https:// www.R-project.org/ [cited 2020. Oct 26].

12. Sondka Z, Bamford S, Cole CG, Ward SA, Dunham I, Forbes SA. The COSMIC Cancer Gene Census: describing genetic dysfunction across all human cancers. Nat Rev Cancer 2018;18:696-705.

13. Durinck S, Spellman PT, Birney E, Huber W. Mapping identifiers for the integration of genomic datasets with the R/ Bioconductor package biomaRt. Nat Protoc 2009;4:1184-91.

14. Athey J, Alexaki A, Osipova E, Rostovtsev A, SantanaQuintero LV, Katneni U, et al. A new and updated resource for codon usage tables. BMC Bioinformatics 2017;18:391.

15. Ellrott K, Bailey MH, Saksena G, Covington KR, Kandoth $\mathrm{C}$, Stewart $\mathrm{C}$, et al.; MC3 Working Group; Cancer Genome Atlas Research Network. Scalable Open Science Approach for Mutation Calling of Tumor Exomes Using Multiple Genomic Pipelines. Cell Syst 2018;6:271-281.e7.

16. Butt AM, Nasrullah I, Tong Y. Genome-wide analysis of codon usage and influencing factors in chikungunya viruses. PLoS One 2014;9:e90905.

17. Wright F. The 'effective number of codons' used in a gene. Gene 1990;87:23-9.

18. Fuglsang A. The 'effective number of codons' revisited. Biochem Biophys Res Commun 2004;317:957-64.

19. Sueoka N. Directional mutation pressure and neutral molecular evolution. Proc Natl Acad Sci USA 1988;85:2653-7.

20. Kyte J, Doolittle RF. A simple method for displaying the hydropathic character of a protein. J Mol Biol 1982;157:105-32.

21. Dana A, Tuller T. The effect of tRNA levels on decoding times of mRNA codons. Nucleic Acids Res 2014;42:9171-81.

22. Gardin J, Yeasmin R, Yurovsky A, Cai Y, Skiena S, Futcher B. Measurement of average decoding rates of the 61 sense codons in vivo. eLife 2014;3:e03735. 
23. Supek F, Miñana B, Valcárcel J, Gabaldón T, Lehner B. Synonymous mutations frequently act as driver mutations in human cancers. Cell 2014;156:1324-35.
24. Sharma Y, Miladi M, Dukare S, Boulay K, CaudronHerger M, Groß M, et al. A pan-cancer analysis of synonymous mutations. Nat Commun 2019;10:2569.

Conflicts of interest.-The authors certify that there is no conflict of interest with any financial organization regarding the material discussed in the manuscript.

Authors' contributions.-Daniele Pepe gave substantial contributions to analysis conceptualization and performance; Daniele Pepe and Kim De Keersmaecker contributed to manuscript draft and revision. All authors read and approved the final version of the manuscript.

History.-Article first published online: October 26, 2020. - Manuscript accepted: October 20, 2020. - Manuscript revised: September 9, 2020. - Manuscript received: May 27, 2020. 